\title{
Atrial fibrillation as a preoperative risk factor predicts long-term mortality in elderly patients without heart failure and undergoing hip fracture surgery
}

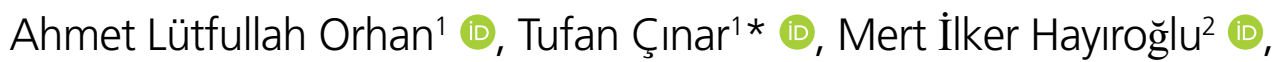 \\ Vedat Çiçek ${ }^{1}$ (D), Murat Selçuk ${ }^{1}$ (D), Selami Doğan ${ }^{1}$ (D), Suha Asal ${ }^{1}$ (1), \\ Samet Yavuz ${ }^{1}$, Serdar Orhan³ (i), Nurgül Keser ${ }^{1}$ (i)
}

\begin{abstract}
SUMMARY
OBJECTIVE: Patients with atrial fibrillation (AF) constitute a significant portion of hip fracture patients, and both diseases tend to present more frequently in older age. Our goal was to evaluate the long-term mortality of patients with AF who were free from heart failure undergoing hip fracture surgery.

METHODS: This observational, retrospective study was done in a single research and training hospital setting. Hospital electronic health record data, National Health Registry data, and National Death Registry System data for 233 consecutive patients who were above 65 years of age and were planned to undergo surgery for hip fracture were retrieved and analyzed. An experienced cardiologist evaluated the patients prior to surgery. Each member of the research cohort was categorized into one of the two groups based on their survival status (survivor and non-survivor groups).

RESULTS: Of the 233 cases, 89 (38.2\%) who were included in the investigation died during the follow-up period. The median long-term follow-up period was 34 (12-42) months. The frequency of AF was significantly higher in the non-survivor group. In multivariable Cox regression analysis, AF (HR: 2.195, 95\% Cl 1.365-3.415, p<0.001), advanced age, and blood urea level were determined as independent predictors for all-cause long-term mortality.

CONCLUSIONS: AF is an independent predictor for long-term death in hip fracture cases above 65 years of age who were free from heart failure.

KEYWORDS: Atrial fibrillation. Hip fractures. Mortality. Long-term effects.
\end{abstract}

\section{INTRODUCTION}

The prevalence of atrial fibrillation (AF) significantly increases as people get older, making it the common arrhythmia in people above 65 years of age ${ }^{1}$. In those above 65 and 80 years of age, the prevalence is roughly $5 \%$ and $10 \%$, respectively. Moreover, approximately $70 \%$ of cases suffering from $\mathrm{AF}$ are between the ages of 65 and 85 years ${ }^{2}$. Prior studies reported that AF was linked with a 1.9-fold increase in death rate during the long-term follow-up even after adjusting for coexisting cardiovascular diseases ${ }^{3}$. Additionally, even though $\mathrm{AF}$ is not included in the current perioperative risk prediction models for noncardiac surgery risk assessment, a large database registry clearly

\footnotetext{
'Health Sciences University, Sultan II, Abdülhamid Han Training and Research Hospital, Department of Cardiology - Istanbul, Turkey. ${ }^{2}$ Health Sciences University, Dr. Siyami Ersek Training and Research Hospital, Department of Cardiology - Istanbul, Turkey.

${ }^{3}$ Health Sciences University, Sultan II, Abdülhamid Han Training and Research Hospital, Department of Orthopedic - Istanbul, Turkey.

*Corresponding author: drtufancinar@gmail.com

Conflicts of interest: the authors declare there is no conflicts of interest. Funding: none.

Received on July 19, 2021. Accepted on August 19, 2021.
} 
reported that patients with AF had a higher risk of perioperative and postoperative complications than those with coronary artery disease ${ }^{4}$.

Hip fractures that are commonly observed in elderly people are described as skeletal system injuries. Remarkably, when compared to those without a hip fracture, older people with a hip fracture have a 5- to 8-fold higher chance of dying during the first 3 months ${ }^{5}$, and this increased risk of death persists for around 10 years $^{6}$. Moreover, elderly people operated for hip fractures have a shorter life expectancy than their counterparts in the same age group 7 .

The presence of AF in elderly patients suffering from hip fracture is a common clinical scenario, and AF shares a number of risk factors with hip fracture, including diabetes, elder age, hypertension, and heart failure (HF). Even though some studies show that both AF and $\mathrm{HF}$ are associated with hip fracture due to an increase in the incidence of falls resulting from stroke and medication ${ }^{8}$, few studies evaluate the effect of $\mathrm{AF}$ on long-term mortality in elderly cases without HF and who underwent surgery for hip fracture. As a consequence, the main goal of this investigation was to evaluate the impact of $\mathrm{AF}$ on long-term mortality in elderly patients above 65 years of age with normal left ventricle function and who were recently operated for hip fracture.

\section{METHODS}

\section{Study cohort}

This study was planned as a single-center, observational, and retrospective cohort investigation. Between January 2017 and December 2019, the data of patients who were above 65 years of age and for whom a preoperative cardiology consultation was requested before the surgery due to hip fracture were analyzed. Patients who died during the index hospitalization and who were diagnosed with paroxysmal AF and HF were excluded from the study. Finally, 233 consecutive elderly cases undergoing surgery for hip fracture were analyzed. The use of medications, the presence of comorbidities, diabetes, hypertension, hyperlipidemia, previous cerebrovascular accident, and demographic features such as age were gathered from the electronic medical files. Additionally, pre-op laboratory values, including hemoglobin level, platelet count, blood creatinine, and thyroid-stimulating hormone levels, were analyzed. AF was defined as the absence of $\mathrm{P}$ waves, the presence of fibrillatory waves, and irregularly irregular QRS complexes on surface electrocardiography (ECG) that failed to self-terminate within 7 days. For each patient, the standard surgical procedures, such as closed reduction, open reduction, and hemiarthroplasty, were applied. Our study design was evaluated and approved by the Local Ethics Commission (21/356).

\section{Preoperative evaluation}

All cases were evaluated by an experienced consultant physician before the operation, together with electrocardiography and transthoracic echocardiography within the framework of the recommendations of the relevant guideline of the European Society of Cardiology published in $2014^{9}$. If required, preoperative treatment was also initiated.

\section{Study outcome}

The main purpose of the investigation was the long-term mortality. The median long-term follow-up period was 34 (12-42) months. By using a lifelong and unique national identification number, the data from the National Death Registry System were used to identify long-term mortality.

\section{Statistical analysis}

The Statistical Package for Social Sciences 20.0 software (SPSS Inc., IL, USA) was used to conduct statistical analyses. The Kolmogorov-Smirnov test was applied to determine the normality of the data. Continuous variables were displayed as median (interquartile range). Absolute and relative frequencies were used to depict quantitative data. Either independent Student's t test or Mann-Whitney U test was used to compare the continuous variables. To analyze the categorical data, we utilized either the Pearson's chi-square test or Fisher's exact test. The independent predictors of long-term death were first determined using the univariable Cox regression analysis. Later, those parameters with statistical significance in the univariable Cox regression analysis were entered into a multivariable Cox regression analysis to find the independent predictors of long-term death. To estimate the chance of surviving based on the presence of AF, a Kaplan-Meier cumulative survival curve analysis was used. In all statistical analyses, a $\mathrm{p}<0.05$ was deemed significant.

\section{RESULTS}

A total of 233 patients who were above 65 years of age and were evaluated by an experienced cardiologist preoperatively were included in the study. Each member of the research cohort was categorized into one of the two groups based on their survival status (survivor and non-survivor groups). During the follow-up period, 89 (38.2\%) elderly patients died.

Table 1 displays the demographic properties and previous medications of all cases enrolled in the study. Patients in the non-survivor group were older, and the frequency of AF was significantly higher in this group. However, the distribution 
Table 1. Comparison of demographic and clinical characteristics of patients according to long-term mortality after hip fracture surgery.

\begin{tabular}{|c|c|c|c|}
\hline & Long-term mortality $(-),(n=144)$ & Long-term mortality $(+),(n=89)$ & $\mathrm{p}$-value \\
\hline Age, year & $81(73-86)$ & $86(80-89)$ & $<0.001$ \\
\hline Male gender, n (\%) & $33(22.9)$ & $30(33.7)$ & 0.074 \\
\hline Hypertension, n (\%) & $115(79.9)$ & $64(71.9)$ & 0.165 \\
\hline Diabetes mellitus, n (\%) & $465(31.3)$ & $22(24.7)$ & 0.285 \\
\hline Insulin dependency, n (\%) & $11(7.6)$ & $7(7.9)$ & 0.950 \\
\hline Hyperlipidemia, n (\%) & $15(10.4)$ & $7(7.9)$ & 0.513 \\
\hline COPD, n (\%) & $14(9.7)$ & $15(16.9)$ & 0.114 \\
\hline Dementia, n (\%) & $30(20.8)$ & $27(30.3)$ & 0.104 \\
\hline Cancer, n (\%) & $27(18.8)$ & $13(14.6)$ & 0.411 \\
\hline Coronary artery disease, $\mathrm{n}(\%)$ & $23(16.1)$ & $14(15.7)$ & 0.943 \\
\hline CRF, n (\%) & $13(9.0)$ & $13(14.6)$ & 0.194 \\
\hline CVA, n (\%) & $8(5.6)$ & $13(14.6)$ & 0.021 \\
\hline Atrial fibrillation, n (\%) & $13(9.0)$ & $26(29.2)$ & $<0.001$ \\
\hline \multicolumn{4}{|l|}{ Medical treatment, n (\%) } \\
\hline Psychiatric drug & $38(26.4)$ & $17(19.1)$ & 0.198 \\
\hline Acetylsalicylic acid & $39(27.1)$ & $19(21.6)$ & 0.345 \\
\hline Clopidogrel & $14(9.7)$ & $9(10.1)$ & 0.923 \\
\hline Beta-blocker & $47(32.9)$ & $36(40.9)$ & 0.216 \\
\hline Calcium channel blocker & $37(25.7)$ & $19(21.3)$ & 0.448 \\
\hline ACE inhibitor/ARB & $69(47.9)$ & $40(45.5)$ & 0.715 \\
\hline Spironolactone & $1(0.7)$ & $1(1.1)$ & 1.000 \\
\hline Furosemide & $8(5.6)$ & $10(11.2)$ & 0.121 \\
\hline Warfarin & $8(5.6)$ & $10(11.2)$ & 0.121 \\
\hline NOACs & $5(3.5)$ & $13(14.8)$ & 0.002 \\
\hline Follow-up, months & $24(14-28)$ & $5(2-16)$ & \\
\hline
\end{tabular}

Continuous variables are presented as median (interquartile range) and nominal variables as frequency (\%). COPD: chronic obstructive pulmonary disease; CRF: chronic renal failure; CVA: cerebrovascular accident; ACE: angiotensin converting enzyme; ARB: angiotensin-receptor blocker; NOACs: new oral anticoagulants.

of other comorbid conditions, such as hypertension, diabetes, cerebrovascular accident, chronic obstructive pulmonary disease, coronary artery disease, and cancer, was similar in both groups. In terms of the use of previous medications, consumption of new oral anticoagulants was statistically significantly higher in the non-survivor group.

Table 2 summarizes comparison of the two groups in terms of laboratory and echocardiographic parameters. Except for blood urea and albumin levels, there was no statistically significant difference between the groups in terms of laboratory data. As for the echocardiographic parameters, patients in the non-survivor group had a higher left atrium anteroposterior diameter. Other echocardiography parameters were similar between the two groups.

According to the univariable Cox regression analysis, advanced age, previous cerebrovascular accident, the presence of AF, blood urea and albumin levels, and left atrium anteroposterior diameter on echocardiography were associated with the long-term all-cause death. In the multivariable Cox regression analysis, the presence of AF (HR: $2.195,95 \% \mathrm{CI}$ $1.365-3.415, \mathrm{p}<0.001$ ), advanced age, and blood urea levels were determined as independent predictors for the long-term all-cause mortality in elderly patients above 65 years of age with hip fracture. 
Table 2. Comparison of laboratory and echocardiography parameters of patients according to long-term mortality after hip fracture surgery.

\section{Long-term mortality $(-),(n=144) \quad$ Long-term mortality $(+),(n=89) \quad$ p-value}

\begin{tabular}{|c|c|c|c|}
\hline \multicolumn{4}{|l|}{ Laboratory variables } \\
\hline Hematocrit, \% & $34.5(31.6-38.2)$ & $33.6(30.3-37.2)$ & 0.213 \\
\hline Hemoglobin, g/dL & $11.6(10.4-12.6)$ & $11.3(10.2-12.3)$ & 0.433 \\
\hline RDW, \% & $13.4(12.6-14.0)$ & $13.5(12.4-15.0)$ & 0.443 \\
\hline WBC count, cells/ $/ \mu \mathrm{L}$ & $8.7(7.3-11.0)$ & $8.5(6.8-10.8)$ & 0.350 \\
\hline Platelet count, cells $/ \mu \mathrm{L}$ & $209(163-254)$ & $215(172-282)$ & 0.271 \\
\hline MPV, fL & $8.8(7.8-9.7)$ & $8.5(7.5-9.7)$ & 0.128 \\
\hline $\mathrm{PCT}, \%$ & $0.19(0.14-0.24)$ & $0.18(0.14-0.27)$ & 0.805 \\
\hline Creatinine, mg/dL & $1.0(0.8-1.2)$ & $1.0(0.8-1.3)$ & 0.339 \\
\hline Urea, mg/dL & $42(34-57)$ & $51(38-78)$ & $<0.001$ \\
\hline $\mathrm{TSH}, \mathrm{nmol} / \mathrm{L}$ & $1.3(0.9-1.7)$ & $1.2(0.5-1.9)$ & 0.739 \\
\hline $\mathrm{T} 4, \mathrm{nmol} / \mathrm{L}$ & $0.9(0.8-1.1)$ & $1.0(0.8-1.1)$ & 0.292 \\
\hline AST, U/L & $22(17-28)$ & $22(17-31)$ & 0.854 \\
\hline ALT, U/L & $16(12-22)$ & $16(12-25)$ & 0.359 \\
\hline Glucose, mg/dL & $121(103-147)$ & $118(97-142)$ & 0.327 \\
\hline $\mathrm{CRP}, \mathrm{mg} / \mathrm{dL}$ & $56(21-89)$ & $46(22-90)$ & 0.915 \\
\hline Troponin, ng/mL & $9.6(5.5-27.0)$ & $21.5(11.0-47.0)$ & 0.003 \\
\hline Albumin, mg/dL & $33(31-37)$ & $30(27-33)$ & $<0.001$ \\
\hline Lymphocytes, cells/ $\mu \mathrm{L}$ & $1.5(1.1-2.0)$ & $1.4(0.9-1.8)$ & 0.055 \\
\hline \multicolumn{4}{|l|}{ Echocardiography variables } \\
\hline Ejection fraction, \% & $61(60-62)$ & $61(60-62)$ & 0.582 \\
\hline LAAP diameter, mm & $37(36-39)$ & $38(36-43)$ & 0.003 \\
\hline LVEDD, mm & $48(45-51)$ & $48(46-50)$ & 0.701 \\
\hline LVESD, mm & $36(34-38)$ & $36(34-38)$ & 0.692 \\
\hline
\end{tabular}

Continuous variables are presented as median (interquartile range) and nominal variables as frequency (\%). RDW: red cell distribution width; WBC: white blood cell; MPV: mean platelet volume; PCT: plateletcrit; TSH: thyroid-stimulating hormone; AST: aspartate aminotransferase; ALT: alanine aminotransferase; CRP: c-reactive protein; LAAP: left atrium anteroposterior; LVEDD: left ventricle end-diastolic volume; LVESD: left ventricle end-systolic volume.

The Kaplan-Meier cumulative survival curve analysis revealed that AF significantly reduced the long-term survival rate in elderly cases above 65 years of age with hip fracture, as shown in Figure 1 (log-rank chi-square test: 14.408, p<0.001).

\section{DISCUSSION}

The incidence of hip fracture increases dramatically in persons above 65 years of age 5 . Surgical treatment is the recommended option for most patients. Even when surgically managed, outcomes are not perfect for these patients ${ }^{5,6}$. Advanced age in this group is commonly associated with increased frailty and worse surgical and survival outcomes ${ }^{10}$. In this age group, comorbidities such as diabetes mellitus and hypertension are more prevalent. As stated in our study, advanced age and impaired renal function are independent predictors for long-term mortality after hip fracture. However, the presence of diabetes mellitus and hypertension was not found to have an effect on long-term mortality. Besides, HF is a known independent predictor of mortality in hip fracture cases ${ }^{10}$. However, there is a scarcity of data for mortality in patients without $\mathrm{HF}$ and with AF specifically. To our knowledge, long-term mortality of these patients has not been studied yet.

One of the most common comorbidities in this age group is AF. In our study, 39 (16\%) of 233 patients had an AF. This is consistent with the current literature, which puts the prevalence of AF at approximately $12-15 \%{ }^{11}$. Previously, in-hospital 


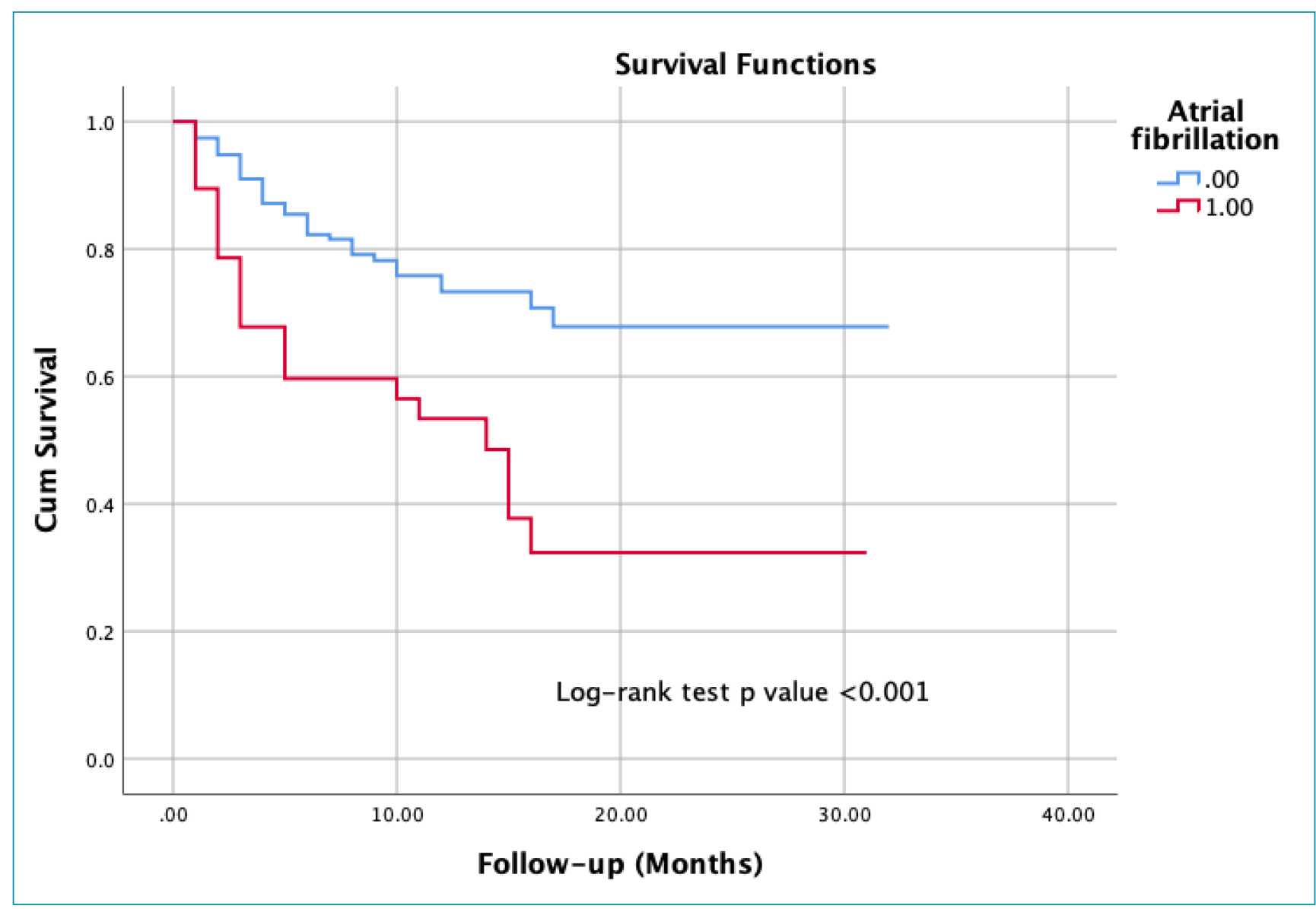

Figure 1. A Kaplan-Meier curve analysis illustrating the cumulative long-term survival function of hip fracture cases based on the presence of atrial fibrillation.

and 1-year mortality for hip fracture in patients with AF had been studied. Neuhaus et al. evaluated the data in the National Hospital Discharge Survey cohort and concluded that AF was a predictor of in-hospital death in hip fracture patients with an odds ratio $(\mathrm{OR})$ of $2.00(\mathrm{p}<0.001)$, and the presence of AF predicted adverse events with an OR of $1.26(\mathrm{p}<0.001)^{12}$. Adunsky et al. studied 1,114 consecutive hip fracture patients and divided the cohort into three groups: sinus rhythm, paroxysmal AF, and chronic AF. At the end of 1 year, only the chronic AF group had an increased mortality rate, with an HR of $1.786^{13}$. In this study, we expanded the current knowledge by demonstrating that AF was also linked with increased risk of death during the long-term follow-up.

Long-term mortality in these patients may be stemming from a myriad of different causes. Orthopedic surgeons may hold back effective anticoagulation in favor of decreasing the hemorrhage risk. However, this decision may be responsible for the increase in mortality by way of ischemic strokes. Additionally, AF increases the risk of stroke 5-fold ${ }^{14}$. Even with effective anticoagulation, thrombi may form in the left atrial appendix. Microthrombi from the left atrium may cause emboli to the brain. Remarkably, hip fracture patients who have stroke are at an increased risk for long-term mortality ${ }^{15}$.

There are multiple guidelines and risk scores designed for preoperative evaluation of patients for noncardiac surgery, the Revised Cardiac Risk Index ${ }^{16}$ (RCRI, Lee's Score) and the American College of Surgeons National Surgical Quality Improvement Program (ACS NSQIP) Index ${ }^{17}$ being the most prominently known ones. Both these scoring indexes do not include $\mathrm{AF}$ as a risk factor. Together with our findings, clinicians using these scoring indexes may modify their interpretation of their results taking AF into account.

\section{Study limitations}

Our study was designed in a retrospective manner, conducted in a single center. Even though we conducted multivariate analyses, frailty indices, such as Charleston morbidity index, assessing the patient's frailty specifically, which can have an effect on hip fracture and all-cause mortality, were not assessed. We only recruited patients with chronic $\mathrm{AF}$, and patients with paroxysmal $\mathrm{AF}$ were 
excluded. Missing physician appointments may play a role in mortality by suboptimal management of both diseases during the follow-up period. Also, some of the patients might even have discontinued their oral anticoagulant regimes by themselves.

\section{CONCLUSIONS}

Chronic $\mathrm{AF}$ is an independent predictor of long-term death in hip fracture patients who were free from HF. Clinicians responsible for the management of hip fracture patients should also manage AF optimally for further reducing the long-term adverse events.

\section{AUTHORS" CONTRIBUTIONS}

ALO: Conceptualization, Formal analysis, Supervision, Writingoriginal draft, Writing-review \& editing. TÇ: Conceptualization, Methodology, Supervision, Writing - original draft, Writing review \& editing. MIH: Formal analysis, Validation, Writing - review \& editing. VÇ: Data curation, Funding acquisition, Resources. MS: Data curation, Funding acquisition, Resources. SD: Data curation, Funding acquisition. SA: Data curation, Funding acquisition. SY: Data curation, Funding acquisition. SO: Project administration, Visualization. NK: Supervision, Validation.

\section{REFERENCES}

1. Falk RH. Atrial fibrillation. N Engl J Med. 2001;344(14):106778. https://doi.org/10.1056/NEJM200104053441407. Erratum in N Engl J Med. 2001;344(24):1876

2. Murphy NF, Simpson CR, Jhund PS, Stewart S, Kirkpatrick M, Chalmers J, et al. A national survey of the prevalence, incidence, primary care burden and treatment of atrial fibrillation in Scotland. Heart. 2007;93(5):606-12. https://doi.org/10.1136/ hrt.2006.107573

3. Benjamin EJ, Wolf PA, D'Agostino RB, Silbershatz H, Kannel $W B$, Levy D. Impact of atrial fibrillation on the risk of death: the Framingham Heart Study. Circulation. 1998;98(10):94652. https://doi.org/10.1161/01.cir.98.10.946

4. van Diepen S, Bakal JA, McAlister FA, Ezekowitz JA. Mortality and readmission of patients with heart failure, atrial fibrillation, or coronary artery disease undergoing noncardiac surgery: an analysis of 38047 patients. Circulation. 2011;124(3):289-96. https://doi.org/10.1161/CIRCULATIONAHA.110.011130

5. Phy MP, Vanness DJ, Melton 3rd L, Long KH, Schleck CD, Larson $D R$, et al. Effects of a hospitalist model on elderly patients with hip fracture. Arch Intern Med. 2005;165(7):796-801. https://doi.org/10.1001/archinte.165.7.796

6. Haentjens P, Magaziner J, Colón-Emeric CS, Vanderschueren $D$, Milisen K, Velkeniers B, et al. Meta-analysis: excess mortality after hip fracture among older women and men. Ann Intern Med. 2010;152(6):380-90. https://doi.org/10.7326/00034819-152-6-201003160-00008

7. Vestergaard P, Rejnmark L, Mosekilde L. Loss of life years after a hip fracture. Acta Orthop. 2009;80(5):525-30. https://doi. org/10.3109/17453670903316835

8. Hung $C Y$, Wu TJ, Wang KY, Huang JL, Loh EW, Chen YM, et al. Falls and atrial fibrillation in elderly patients. Acta Cardiol Sin. 2013;29(5):436-43. PMID: 27122741

9. Kristensen SD, Knuuti J, Saraste A, Anker S, Bøtker HE, Hert S, et al. 2014 ESC/ESA Guidelines on non-cardiac surgery: cardiovascular assessment and management: The Joint Task Force on non-cardiac surgery: cardiovascular assessment and management of the European Society of Cardiology (ESC) and the European Society of Anaesthesiology (ESA). Eur Heart J. 2014;35(35):2383-431. https://doi.org/10.1093/eurheartj/ ehu282

10. Kistler EA, Nicholas JA, Kates SL, Friedman SM. Frailty and short-term outcomes in patients with hip fracture. Geriatr Orthop Surg Rehabil. 2015;6(3):209-14. https://doi. org/10.1177/2151458515591170

11. Abu-Assi R, Campbell J, Bacchi S, Gill TK, George D, Chehade M. Association between atrial fibrillation and hip fractures and the implications for hip fracture patients: a systematic review. ANZ J Surg. 2020;90(4):448-53. https://doi.org/10.1111/ ans. 15460

12. Neuhaus V, King J, Hageman MG, Ring DC. Charlson comorbidity indices and in-hospital deaths in patients with hip fractures. Clin Orthop Relat Res. 2013;471(5):1712-9. https://doi. org/10.1007/s11999-012-2705-9

13. Adunsky A, Arad M, Koren-Morag N, Fleissig Y, Mizrahi EH. Increased 1-year mortality rates among elderly hip fracture patients with atrial fibrillation. Aging Clin Exp Res. 2012;24(3):233-8. https://doi.org/10.1007/BF03325251

14. Pisters R, Lane DA, Marin F, Camm AJ, Lip GYH. Stroke and thromboembolism in atrial fibrillation. Circ J. 2012;76(10):2289304. https://doi.org/10.1253/circj.cj-12-1036

15. Dennis MS, Lo KM, McDowall M, West T. Fractures after stroke: frequency, types, and associations. Stroke. 2002;33(3):728-34. https://doi.org/10.1161/hs0302.103621

16. Ford MK, Beattie WS, Wijeysundera DN. Systematic review: prediction of perioperative cardiac complications and mortality by the revised cardiac risk index. Ann Intern Med. 2010;152(1):26-35. https://doi.org/10.7326/0003-4819-1521-201001050-00007

17. Bilimoria $K Y$, Liu $Y$, Paruch $J L$, Zhou L, Kmiecik TE, Ko $C Y$, et al. Development and evaluation of the universal ACS NSQIP surgical risk calculator: a decision aid and informed consent tool for patients and surgeons. J Am Coll Surg. 2013;217(5):833-42.e1-3. https://doi.org/10.1016/j. jamcollsurg.2013.07.385 